\title{
Infrared spectroscopy study of the ethanolic leaf extracts of Artocarpus heterophyllus and Litchi chinensis
}

\begin{abstract}
A phytochemical study has been conducted using infrared spectroscopy to distinguish different chemical moieties present in leaves of Artocarpus heterophyllus (Jack fruit) and Litchi chinensis (Litchi). Both of these trees are widely known in the world and have various pharmacological activities. However, both leaf extracts were analyzed using FTIR and interpreted from the obtained spectrum. Both leaves are supposed to have $-\mathrm{OH},-\mathrm{NH}_{2}$ groups as they give a wide peak in higher region wavelength (3400$3200 \mathrm{~cm}^{1}$ ). Meta-substituted aromatic ring and imides may be present in litchi leaf extract as peaks were found in $850-700 \mathrm{~cm}^{-1}$ and near $1700 \mathrm{~cm}^{-1}$ respectively, but not in jackfruit leaf. While phenol group is present in the spectrum of jackfruit leaf extract only. Asymmetric methyl group is present in both leaf extract as gives absorption in the $2957-2851 \mathrm{~cm}^{-1}$ region.
\end{abstract}

Volume I Issue 5 - 2017

\author{
Saifuddin AHM,' Ruhul Mahbub MD,' Sharmin \\ Akter Mst,' Jannatul Fardous ${ }^{2}$ \\ 'Department of Pharmacy, Jahangirnagar University, Bangladesh \\ ${ }^{2}$ Department of Pharmacy, Commilla University, Bangladesh
}

Correspondence: AHM Saifuddin, Department of Pharmacy, Jahangirnagar University, Savar, Dhaka- 1342, Bangladesh, Email ahm.saifuddinII@gmail.com

Received: December 01, 2017 | Published: December 13, 2017

Keywords: phytochemical study, leaf extracts, ftir, spectrum, asymmetric methyl group

\section{Abbreviations: FT, fourier transform; IR, infrared; FTIR, fou-} rier transforms infrared

\section{Introduction}

Infrared spectroscopy is a standard method of analytical pharmacy and chemistry which provides the images of vibration of atoms of compound Dole et al. ${ }^{1}$ Fourier transform (FT) infrared (IR) spectrometers were commercially introduced in 1970. They were characterized by peculiar analytical performances that mainly consisted in the use of trace sample (down to 100ng), high throughput measurements without sample destruction and with minimal sample preparation Bellisola et al. ${ }^{2}$ Artocarpus heterophyllus (Jack-fruit), belonging to family 'Moraceae' Taylor et al. ${ }^{3}$ It is known as 'Kathal' in Bangladesh and found in most of the part in Bangladesh. The young fruits are acrid, astringent, carminative and tonic. The ripe fruits are sweet, cooling, laxative, an aphrodisiac and tonic. The bark is useful in fever, boils, wounds, skin diseases. The jackfruit is considered an invasive species in Brazil, especially in the Tijuca Forest National Park in Rio de Janeiro Lakheda et al. ${ }^{4}$

Litchi chinensis (Litchi) is a tropical and subtropical fruit native to South East Asian countries and now widely cultivated throughout the world Duan et al..$^{5}$ Litchi or lychee, a fruit with a rough brown pericarp surrounding a white flesh (aril), is popular for its delicious taste and possible health benefits Jiang et al. ${ }^{6}$ Various biologically active constituents are present in different parts of lychee (leaves, flower, fruit, pericarp and seed). These compounds are reported to exhibit several pharmacological activities Taak et al. ${ }^{7}$

\section{Materials and method}

\section{Collection of plant material}

The jackfruit and litchi leaves were collected from Jahangirnagar University campus, Bangladesh. The leaves were dried and crushed manually with the wooden arrangement and made in powder form.

\section{Extraction}

The plant materials were sun-dried first and then, dried in an oven at reduced temperature $(<700 \mathrm{C})$ to make suitable for grinding. The powdered plant materials were submerged in sufficient volume of ethanol in an air-tight flat-bottomed container for seven days, with occasional shaking and stirring. The extracts were then filtered and dried in an electrical water bath. Fourier transforms infrared (FTIR) spectroscopy: Fourier transform infrared (FTIR) spectra were recorded using FTIR spectrophotometer (IR prestige- 21, Shimadzu). The samples (JFL_Extract and Lit_Extract) were previously ground and mixed thoroughly with potassium bromide (an infrared transparent matrix) in a ratio of 1:100. The $\mathrm{KBr}$ discs were prepared by compressing the powders at a pressure of 6 tons for $5 \mathrm{~min}$ in a hydraulic press (Shimadzu, Japan). Twenty-five scans for Artocarpus heterophyllus and thirty-eight scans for Litchi chinensis were obtained at a resolution of $1 \mathrm{~cm}^{-1}$, from 4000 to $500 \mathrm{~cm}^{-1}$.

\section{Results and discussion}

The spectrum (Figure 1) obtained from FTIR. It is found that absorption vibration took place in the whole infrared region $\left(4000 \mathrm{~cm}^{-}\right.$ ${ }^{1}$ to $\left.667 \mathrm{~cm}^{-1}\right)$. Most of the absorption peaks are found in the frequency of $1700 \mathrm{~cm}^{-1}$ to $1000 \mathrm{~cm}^{-1}$ for both of the extracts. This indicates the presence of nitro compounds, esters, ethers, primary, secondary and tertiary alcohols generally Sharma et $a .^{8}$ From the JFL_Extract spectrum, the appearance of the medium band in $3500-3300 \mathrm{~cm}^{-1}$ regions, we can assume the presence of $-\mathrm{OH},-\mathrm{NH}_{2}$ group. Besides this band on $3301 \mathrm{~cm}^{-1}$ indicates the existence of alkyne stretches $(\equiv \mathrm{C}-\mathrm{H})$ with strong intensity Pavia et al. ${ }^{9}$ Combination and overtone absorption occurs in the $2957-2851 \mathrm{~cm}^{-1}$ region. This absorption may be due to $\mathrm{C}-\mathrm{H}$ stretching from methyl or methylene groups. Absorption is asymmetric as absorption took place in higher wavelength Sharma et al. ${ }^{8}$ Absorption peaks in the fingerprint region (the region below $1500 \mathrm{~cm}^{-1}$ ) here indicates the presence of some different functional groups viz. alcohols, esters, lactones, anhydrides Pavia et al. ${ }^{9}$ 
Absorption in this region particularly $1350^{-1} 000 \mathrm{~cm}^{-1}$ takes place due to $\mathrm{C}-\mathrm{O}$ stretching. Peaks in this region also recommend existence of phenol group here as phenols absorb near $1200 \mathrm{~cm}^{-1}$ Sharma et al. ${ }^{8}$ and such peaks are present in the spectrum $\left(1166 \mathrm{~cm}^{-1}\right.$ and $\left.1224 \mathrm{~cm}^{-1}\right)$.

Spectrum for Lit_Extract is quite similar to the previous one. Presence of $-\mathrm{OH}$, $-\mathrm{NH} 2$ group is possible here also as a wide peak is present in $3404 \mathrm{~cm}^{-1}$ region with medium intensity. An interesting finding in this spectrum is that combination and overtone absorption occurs in a $2851-2958 \mathrm{~cm}^{-1}$ region which is same as the jackfruit leaf extract. So methyl or methylene groups are present here as the result of $\mathrm{C}-\mathrm{H}$ stretching. Numbers of absorption peaks are higher in fingerprint region, and absorption took place in $1500-667 \mathrm{~cm}^{-1}$ wavelength. Absorption below $1000 \mathrm{~cm}^{-1}$ indicates the presence of aromatic ring and also is very useful for identifying the type of substitution in the aromatic ring Sharma et al. ${ }^{8}$ In this spectrum absorption peaks in $668.3 \mathrm{~cm}^{-1}, 720.4 \mathrm{~cm}^{-1}$ and $830.3 \mathrm{~cm}^{-1}$ are found, and we can assume that meta-substituted benzene ring is present. Because absorption in 850 $710 \mathrm{~cm}^{-1}$ and $750-700 \mathrm{~cm}^{-1}$ recognizes meta-substituted compounds and mono-substitution of benzene ring respectively. Esters, aldehydes, ketones, imides may be present in litchi leaf due to absorption in 1900${ }^{1} 650 \mathrm{~cm}^{-1}$ region as a result of $\mathrm{C}=\mathrm{O}$ stretching. Presence of imides is possible, as several two strong bands (doublet) found near $1700 \mathrm{~cm}^{-1}$ wavelength Sharma et al. ${ }^{8}$

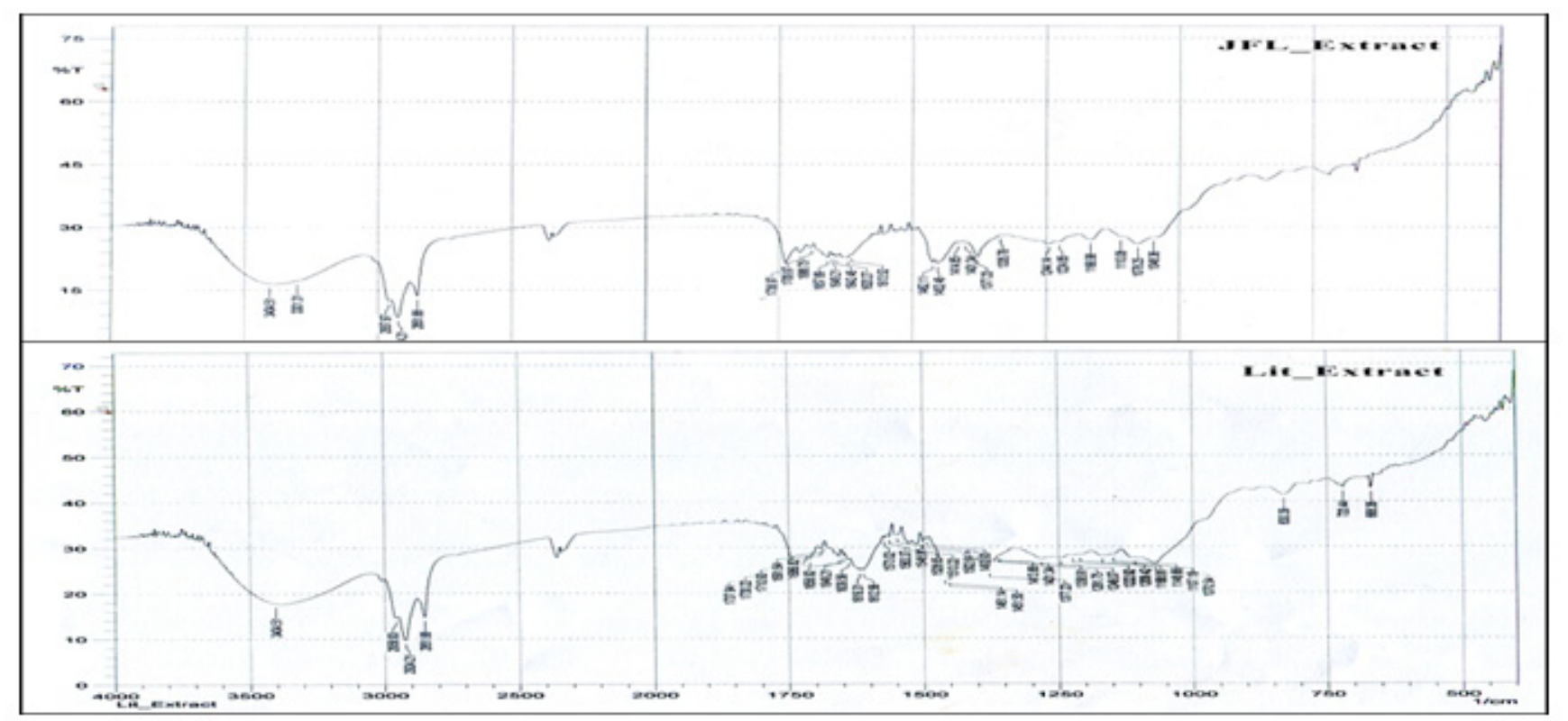

Figure I FTIR spectrograms of, (A), Jack fruit leaves (JFL_Extract); (B), Litchi leaves extract (Lit_Extract).

\section{Conclusion}

From the above discussion and spectrum analysis, it is clear that different functional groups are present in both jack-fruit and litchi leaf. Presence of phenol groups in jackfruit leaf extract emphasizes its antioxidant properties and its use for different therapeutic purposes, e.g., fever, wound healing, skin diseases. Moreover, the substituted aromatic ring structure is present in litchi leaf while absent in jackfruit leaf as per spectrum analysis. Further study on both of these leaf constituents may help to discover a new molecule for medicinal use.

\section{Acknowledgments}

None.

\section{Conflicts of interest}

Author declares that there is no conflict of interest.

\section{References}

1. Dole MN, Patel PA, Sawant SD, et al. Advance applications of fourier transform infrared spectroscopy. International Journal of Pharmaceutical Sciences Review and Research. 2011;7(2):159-166.
2. Bellisola G, Sorio C. Infrared spectroscopy and microscopy in cancer research and diagnosis. Am J Cancer Res. 2012;2(1):1-21.

3. Taylor JL. Forest Monks and the Nation-state: An Anthropological and Historical Study in North East Thailand. 1993. 218 p.

4. Lakheda S, Devalia R, Umesh KJ, et al. Anti -inflammatory activity of Artocarpus heterophyllus bark. Der Pharmacia Sinica. 2011;2(2):127-130.

5. Duan X, Wu G, Jiang Y. Evaluation of the antioxidant properties of litchi fruit phenolics in relation to pericarp browning prevention. Molecules. 2007;12(4):759-771.

6. Jiang G, Lin S, Wen L, et al. Identification of a novel phenolic compound in litchi (Litchi chinensis Sonn.) pericarp and bioactivity evaluation. Food Chem. 2013;136:563-568.

7. Taak P, Koul B. Phytochemistry and Pharmacological Properties of Lychee (Litchi chinensis Sonn). Journal of Chemical and Pharmaceutical Research. 2016;8(10):35-48.

8. Sharma RY. Elementary Organic Spectroscopy. 4th ed. India: Chand \& Company Ltd. Publishers; 2007. p. 89-148.

9. Pavia LD, Lampman MG, Kris SG. Introduction to Spectroscopy. 3rd ed. USA: Harcourt College Publishers; 2001. p. 24-75. 